\section{Entwarnung: kein fetales Risiko nach Grippeschutzimpfung in der Schwangerschaft}

\author{
Säuglinge können nur durch eine Impfung während der Schwangerschaft vor \\ Influenza geschützt werden. Bei der Impfung hatten viele Ärzte Bedenken. \\ Studiendaten zeigen jedoch, dass es keinen Grund zur Zurückhaltung gibt.
}

\begin{abstract}
ie Weltgesundheitsorganisation (WHO) empfiehlt schon seit einiger Zeit die Impfung von Schwangeren gegen saisonale Influenza. Die STIKO hat seit 2010 eine entsprechende Empfehlung ausgesprochen. Erst im Rahmen der Influenzapandemie im Jahr 2009 durch das Influenza-A-Virus H1N1 („Schweinegrippe“) wurden die Empfehlungen hinsichtlich der Impfung von Schwangeren geändert. Hintergrund für diese Änderung war, dass diese Form der Influenza bei Schwangeren besonders schwerwiegend verläuft. Vorher wurde bei der Impfung von werdenden Müttern zur Zurückhaltung geraten.

Haberg et al. haben jetzt im Nachhinein die Sicherheit der Impfung gegen Influenza A/H1N1 überprüft. Hierzu standen Daten von 117.374 Schwangerschaften in Norwegen von 2009 und 2010 zur
\end{abstract}

Verfügung. Die fetale Mortalitätsrate betrug 4,9 Todesfälle pro 1.000 Geburten. Während der Pandemie wurden 54\% der Schwangeren in Norwegen im zweiten oder dritten Trimester geimpft.

Die Impfung senkte das Risiko einer Influenza substanziell. Bei Schwangeren, die klinisch an einer Influenza erkrankt waren, war das Risiko eines fetalen Todesfalls fast verdoppelt (Hazard Ratio [HR]: 1,91). Das Risiko eines fetalen Todesfalls bei den Schwangeren mit Impfung war dagegen kleiner (HR: 0,88), jedoch nicht statistisch signifikant.

Die Autoren schlussfolgern, dass bei dem bekannten Risiko, welches durch eine mütterliche Influenza hervorgerufen wird, die Impfung von Schwangeren während einer Influenzapandemie nicht schadet und möglicherweise dem Feten direkt nutzt. Die Impfung sei daher
Schwangeren im zweiten oder dritten Trimester nicht vorzuenthalten.

Håberg SE et al. Risk of fetal death after pandemic influenza virus infection or vaccination. N Engl J Med 2013; 368 333-40

Kommentar: Die Studie zeigt, dass die vor der Schweinegrippe in Deutschland bestehende Zurückhaltung bei der Impfung von werdenden Müttern gegen Influenza nicht begründet war. Seit 2010 ist laut STIKO eine Impfung von Schwangeren gegen Influenza sogar ausdrücklich angeraten. Sie empfiehlt die Impfung im zweiten Schwangerschaftsdrittel, bei erhöhter gesundheitlicher Gefährdung infolge eines Grundleidens (z. B. Asthma, Diabetes)ab dem ersten Schwangerschaftsdrittel [Epidemiologisches Bulletin 31/2010]. Diese Empfehlung bezieht sich auf die Impfung gegen saisonale Influenza. Dem Impfstoff gegen saisonale Influenza sind jetzt Antigene gegen das Influenza-A-Virus H1N1 beigefügt.

Es muss daran erinnert werden, dass nur durch die Impfung der Schwangeren jungen Säuglingen ein Influenzaschutz vermittelt wird, da die jungen Säuglinge selbst zunächst noch nicht gegen Influenza geimpft werden können.

Dr. Harmut Koch

\title{
Frühgeburt erhöht Risiko für spätere psychische Erkrankungen
}

Frühgeburtlichkeit wurde in den vergangenen Jahren vor allem mit der Ätiologie der Schizophrenie in Verbindung gebracht. Theorien einer gestörten Hirnentwicklung als Erkrankungsursache spielen aber auch bei uni- und bipolarer Depression eine Rolle. Eine bevölkerungsbasierte Studie liefert dafür Hinweise.

W eitestgehende Transparenz und große Bevölkerungsregister - der Alptraum jedes deutschen Datenschützers machen es in Skandinavien möglich, Untersuchungen an großen Teilen der Bevölkerung durchzuführen. So konnten Wissenschaftler Daten von über 1,3 Millionen Schweden, die zwischen 1973 und 1986 in Schweden geboren worden waren und im Alter von 16 Jahren und älter im Jahr 2002 in Schweden lebten, aus drei Registern zusammenführen und auswerten. Dabei wurden Daten zu Schwangerschaftsdauer, Geburtsgewicht und -komplikationen sowie APGAR-Index erfasst und mit der Wahrscheinlichkeit für eine spätere stati- onäre psychiatrische Behandlung wegen nicht affektiver Psychosen, unipolarer Depression, bipolarer Störung, Essstörungen, Alkohol- sowie Drogenabhängigkeit in Beziehung gesetzt.

Frühgeburtlichkeit in der 32. bis 36 . Schwangerschaftswoche erhöhte das Risiko für eine spätere stationär behandlungsbedürftige unipolare Depression um $50 \%$, ein noch früherer Geburtszeitpunkt führte fast zu einer Verdreifachung des Depressionsrisikos unabhängig von zahlreichen anderen Einflussfaktoren. Auch ein niedriger APGAR-Index erhöhte das Depressionsrisiko. Ebenso zeigte sich das Risiko für die anderen erfassten psychischen Störungen bei Erwachsenen erhöht, wenn sie als Frühchen zur Welt gekommen, zu klein für ihr Geburtsalter waren oder einen sehr niedrigen APGAR-Index bei Geburt hatten.

Nosarti $C$ et al. Preterm birth and psychiatric disorders in young adult life. Arch Gen Psychiatry 2012; 69: E1-8

Kommentar: Diese Studie unterstreicht die immense Bedeutung von Schwangerschaft und Geburt für die Ätiologie psychischer Erkrankungen auf einer kaum noch anfechtbaren Datengrundlage. Die Ergebnisse stützen nicht nur die in den letzten Jahren diskutierten Hirnentwicklungsmodelle psychischer Störungen, sondern stellen auch die Erblichkeitsschätzungen der psychiatrischen Genetik infrage, da diese auf der Basis von Konkordanzraten bei Zwillingen geschätzt werden. Eineiige Zwillinge haben ein deutlich erhöhtes Frühgeburtlichkeitssrisiko und kommen nahezu immer zu klein für ihr Gestationsalter auf die Welt. Prof. Dr. Helge Frieling 